\title{
Convergence of the Fixed Point Algorithm of Analytical Models of Reliable Internet Protocols (TCP)
}

\author{
Debessay Fesehaye Kassa and Sabine Wittevrongel \\ Department of Telecommunications and Information Processing, \\ Ghent University, Sint-Pietersnieuwstraat 41 \\ B-9000 Gent, Belgium \\ debessayatelin.ugent.be
}

\begin{abstract}
Analytical models are important tools for the performance investigation of the Internet. The literature shows that the fixed point algorithm (FPA) is one of the most useful ways of solving analytical models of Internet performance.

Apart from what is observed in experimental literature, no comprehensive proof of the convergence and uniqueness of the FPA is given. In this paper we show how analytical models of reliable Internet protocols (TCP) converge to a unique fixed point. Unlike previous work in the literature the basic principles of our proof apply to both single and multiple bottleneck networks, to short and long-lived TCP connections and to both Drop Tail and Active Queue Management (AQM) routers. Our proof of convergence is based on a well known fixed point theorem and our uniqueness proof exploits the feedback nature of the reliable protocol.

The paper specifies conditions under which the FPA of analytical models of TCP converges to a unique point. The concepts used in the proof can also be extended to analyze the equilibrium, stability and global uniqueness issues of TCP, other reliable protocols and the Internet as a whole.
\end{abstract}

Keywords: Analytical models, Internet, TCP, fixed point, convergence, equilibrium, stability.

\section{Introduction}

An equation $\mathbf{f}(\mathbf{x})=\mathbf{x}$ which maps a point $\mathbf{x}$ to itself is called a fixed point equation.

Analytical models are important tools for investigating, designing, dimensioning and planning IP (Internet Protocol) networks. The literature (for example see Olsén et al. [10]) shows that the fixed point algorithm (FPA) is one of the most useful ways of solving analytical models of Internet performance. The fixed point methods combine the detailed models describing the behavior of the sources with network models resulting in a compound model. Many analytical models such as [3, 4, 5, 9] use the fixed point method.

Each homogeneous group of (TCP) connections (flows with the same path, the same TCP version, the same packet size ... ) is represented by a TCP sub-model. Each bottleneck link and the associated router traversed by the groups of TCP connections is represented by a network sub-model. 
The TCP sub-models calculate the load offered to the respective network submodels. The network sub-models in turn calculate the loss probability and queueing delay for the corresponding TCP sub-models in an iterative fixed point procedure (FPA). This is analogous to the fact that the packet loss probability and packet delay in the network depend on the sending rate of the sources, and the flow-controlled (TCP) sources adjust their sending rates in response to observed packet loss and packet delay. Hence the usual fixed point elements (entries) used in solving analytical models of TCP performance are the packet loss probability $P_{L}$, the average queue length $E_{N}$ from which the queueing delay and the RTT (round trip time - the time from the start of packet transmission until the reception of its acknowledgement) are calculated and the load offered by the TCP connections $\Lambda$.

In light of the above discussion, the main contributions of this paper are the following.

- Convergence and Uniqueness Proof: Proof of the convergence of the FPA based on a well established theorem (used in Nash Equilibrium theory) and proof of the uniqueness of the fixed points based on the feedback nature of the reliable protocol are given. Unlike some previous works ( [9,3]) our elegant proofs apply to both single and multi-bottleneck networks, to short and long-lived connections and to AQM and Drop Tail routers at different network scenarios.

- Specification of the Conditions for Convergence and Uniqueness: While proving the convergence and uniqueness we also specify the conditions under which the FPA of analytical models of reliable Internet protocols like TCP converges to a unique point. None of the proofs in the literature ( [9,3]) has explicitly specified these conditions.

- Simplicity: Unlike the few previous works, this paper presents simple and accurate proofs with minimal assumptions.

- Extendibility: Unlike the previous works, an extension of our robust proofs which are based on well known concepts allows us to easily analyze the equilibrium, stability and global uniqueness issues of TCP, other reliable Internet protocols and the Internet as a whole.

The rest of the paper is organized as follows. In sections 2 and 3 we show how the FPA converges to a unique fixed point. We give the summary and work in progress in section 4.

\section{Existence of the Fixed Point}

There are several fixed point theorems based on continuous functions [2] and discontinuous functions [6]. We use the Brouwer's fixed point theorem (see [2]) which is based on continuity of the fixed point function to show the existence of the fixed points.

To prove the convergence of the two-dimensional FPA of analytical models of TCP performance for a single bottleneck link using the Brouwer's fixed point theorem it suffices to show that a continuous function $\mathbf{f}$ exists in a non empty compact convex set or in a closed $n$-ball or equivalent such that

$$
\mathbf{f}\left(P_{L}, E_{N}\right)=\left(P_{L}, E_{N}\right) .
$$




\subsection{The Compact and Convex Set (Region) Where the FPA Is Carried Out}

The packet loss probability value $P_{L}$ is between 0 and 1 and the mean buffer occupancy value $E_{N}$ is between 0 and $K$ where $K-1$ is the router buffer capacity. These two intervals form a non empty compact convex set of points (plane) in which the fixed point procedure is carried out.

We next derive the function $\mathbf{f}$ and show how and why it becomes continuous.

\subsection{The Continuous Fixed Point Function}

To derive the fixed point Equation 1 and show that it is continuous, we will first use well known formulas for the derivation of the load that the TCP sub-models offer to the network sub-models. We then prove that these formulas are continuous and conclude that the formula for the throughput (load offered by a TCP connection) is continuous. We will also use the packet loss probability and queuing delay as given by the $M / M / 1 / K$ queuing system for the network sub-model and explain that these functions are continuous. Finally we will construct the vector valued fixed point function given in Equation1 and show that it is continuous.

The formulas for the TCP sub-models and their continuity. TCP connections adjust the load they offer to the network as a function of the packet loss probability and queueing delay at the bottleneck router. This relationship is given by the well known PFTK throughput formula ( [11]) for TCP Reno.

Let $P_{L}$ denote the packet loss probability at a bottleneck router. Let $E_{N}$ denote the queue length at the bottleneck router. Let $\Lambda$ denote the load offered by the TCP submodel(s). Let $T_{0}$ denote the TCP initial timeout value in seconds. Let $W_{m}$ denote the maximum TCP window size expressed in packets. Let $W\left(P_{L}\right)$ denote the expected TCP window size as a function of packet loss probability, $P_{L}$. Then [11]

$$
W\left(P_{L}\right)= \begin{cases}\frac{2}{3}+\sqrt{\frac{4\left(1-P_{L}\right)}{3 P_{L}}+\frac{4}{9}} & W\left(P_{L}\right)<W_{m} \\ W_{m} & W\left(P_{L}\right) \geq W_{m} .\end{cases}
$$

Let $Q\left(P_{L}, w\right)$ denote the probability that a loss in a window of size $w$ is a timeout (TO). Then [11]

$$
Q\left(P_{L}, w\right)= \begin{cases}1 & w \leq 3 \\ \frac{\left(1-\left(1-P_{L}\right)^{3}\right)\left(1+\left(1-P_{L}\right)^{3}\left(1-\left(1-P_{L}\right)^{w-3}\right)\right)}{1-\left(1-P_{L}\right)^{w}} & w>3, P_{L} \neq 0 \\ \frac{3}{w} & w>3, P_{L}=0 .\end{cases}
$$

Let $G\left(P_{L}\right)$ denote a polynomial term used in the PFTK formula by

$$
G\left(P_{L}\right)=1+P_{L}+2 P_{L}^{2}+3 P_{L}^{3}+4 P_{L}^{4}+5 P_{L}^{5}+6 P_{L}^{6} .
$$

Let $E[X]$ denote the expected round number when the first packet loss occurs. A round begins when a packet is sent and ends when its ACK arrives. As shown in [11],

$$
E[X]= \begin{cases}W\left(P_{L}\right) & W\left(P_{L}\right)<W_{m} \\ \frac{W_{m}}{4}+\frac{1-P_{L}}{P_{L} W_{m}}+1 & W\left(P_{L}\right) \geq W_{m} .\end{cases}
$$


The throughput of a TCP connection is given by the PFTK formula

$$
\lambda=t\left(P_{L}, R T T\right)=\frac{\frac{1-P_{L}}{P_{L}}+\frac{W\left(P_{L}\right)}{2}+Q\left(P_{L}, W\left(P_{L}\right)\right)}{R T T(E[X]+1)+\frac{Q\left(P_{L}, W\left(P_{L}\right)\right) G\left(P_{L}\right) T_{0}}{1-P_{L}}} .
$$

Substituting Equation 5 into Equation 6 and multiplying the first part of the resulting equation by $\left(1-P_{L}\right) /\left(1-P_{L}\right)$ and the second part by $P_{L} / P_{L}$ yields

$$
\lambda=t\left(P_{L}, R T T\right)=\left\{\begin{array}{c}
\frac{\frac{\left(1-P_{L}\right)^{2}}{P_{L}}+\frac{W\left(P_{L}\right)}{2}\left(1-P_{L}\right)+Q\left(P_{L}, W\left(P_{L}\right)\right)\left(1-P_{L}\right)}{R T T\left(W\left(P_{L}\right)+1\right)\left(1-P_{L}\right)+Q\left(P_{L}, W\left(P_{L}\right)\right) G\left(P_{L}\right) T_{0}}, \\
W\left(P_{L}\right)<W_{m} \\
\frac{1-P_{L}+\frac{W\left(P_{L}\right)}{2}\left(P_{L}\right)+Q\left(P_{L}, W\left(P_{L}\right)\right) P_{L}}{P_{L} R T T\left(\frac{W_{m}}{4}+\frac{1-P_{L}}{P_{L} W_{m}}+2\right)+P_{L} \frac{Q\left(P_{L}, W\left(P_{L}\right) G\left(P_{L}\right) T_{0}\right.}{1-P_{L}}} \\
W\left(P_{L}\right) \geq W_{m} .
\end{array}\right.
$$

$W\left(P_{L}\right)<W_{m}$ implies that

$$
\frac{2}{3}+\sqrt{\frac{4\left(1-P_{L}\right)}{3 P_{L}}+\frac{4}{9}}<W_{m}
$$

so that

$$
P_{L}>\frac{1}{\frac{3}{4} W_{m}^{2}-W_{m}+1} .
$$

The function $W\left(P_{L}\right)$ given in Equation 2 is continuous as

$$
\lim _{P_{L} \rightarrow \frac{3}{\frac{3}{4} W_{m}^{2}-W_{m}+1}} W\left(P_{L}\right)=W_{m}=W\left(\frac{1}{\frac{3}{4} W_{m}^{2}-W_{m}+1}\right) .
$$

Similarly it can be shown that $E[X]$ is a continuous function of $P_{L}$.

It can be shown using L'Hopital's rule that the function $Q\left(P_{L}, W_{m}\right)$ described in Equation 3 is a continuous function of $P_{L}$. The function $Q\left(P_{L}, W\left(P_{L}\right)\right)$ is also continuous as $W\left(P_{L}\right)$ is continuous. The polynomial function $G\left(P_{L}\right)$ given by Equation 4 is also continuous.

Besides the continuity of $\lambda=t\left(P_{L}, R T T\right)$ is not affected by the value of $R T T$ which is greater than 0 .

Therefore the function $\lambda=t\left(P_{L}, R T T\right)$ of persistent TCP Reno connections given by Equation 7 is continuous since a combination and composition of continuous functions is continuous at all appropriate points. For other TCP implementations with Drop Tail and RED the throughput formulas given in [7] can be used and the continuity can be similarly shown.

Using similar arguments of the above formulas of persistent TCP connections, the continuity of the square root formula for the rate of non-persistent TCP flows given in [1] can be shown. Any point of discontinuity can be removed by re-defining the function.

We next discuss the continuity of the network sub-model formulas. 
The formulas for the network sub-models and their continuity. The network submodel which focuses on the IP network receives the average traffic load $\Lambda$ packets/sec collectively offered by the TCP sub-model(s). The network sub-model $(M / M / 1 / K)$ with a router buffer capacity of $K-1$ packets and a link capacity of $C$ packets/sec (the load $\rho=\Lambda / C$ ) is used to compute the loss probability $P_{L}$ and the expected number $E_{N}$ of customers in the queueing system. The queueing delay part of the $R T T$ is calculated from $E_{N}$ for the TCP sub-models.

The $M / M / 1 / K$ queueing system yields a closed form formula for the packet loss probability and queue length. A simple way of accounting for the burstiness of TCP traffic using the $M / M / 1 / K$ is shown in [9] so that the closed form expressions of $P_{L}$ and $E_{N}$ still hold.

Using L'Hopital's rule the quantities $P_{L}$ and $E_{N}$ can be shown to be continuous functions $h_{1}(\Lambda)$ and $m(\Lambda)$. This implies that $R T T$ which is a continuous function $u\left(E_{N}\right)$ is also a continuous function $h_{2}(\Lambda)$. If there are $N$ TCP connections, the total load offered $\Lambda=N \lambda=N t\left(P_{L}, R T T\right)=g\left(P_{L}, R T T\right)$ for some continuous function $g$.

The fixed point formula and its continuity. From the above arguments the fixed point equation used in modeling TCP is given by

$$
\begin{aligned}
\left(P_{L}, E_{N}\right) & =\left(h_{1}(\Lambda), m(\Lambda)\right)=\left(h_{1}\left(g\left(P_{L}, R T T\right), m\left(g\left(P_{L}, R T T\right)\right)\right)\right. \\
& =\left(h_{1}\left(g\left(P_{L}, u\left(E_{N}\right)\right), m\left(g\left(P_{L}, u\left(E_{N}\right)\right)\right)\right)=\left(f_{1}\left(P_{L}, E_{N}\right), f_{2}\left(P_{L}, E_{N}\right)\right)\right. \\
& =\mathbf{f}\left(P_{L}, E_{N}\right) .
\end{aligned}
$$

Theorem 2.1 The function $\mathbf{f}$ given in Equation 11 above is continuous.

Proof. The functions, $h_{1}, m, u$, and $g$ are all shown to be continuous in the preceding sections. Hence the functions $f_{1}$ and $f_{2}$ which are compositions of continuous functions are also continuous. This implies that the vector valued fixed point function $\mathbf{f}$ given by Equation 11 is a continuous function.

Now by the Brouwer's fixed point theorem the function $\mathbf{f}$ given by Equation 11 has a fixed point in the non empty compact convex set explained in section 2.1. We next show that this fixed point is unique.

\section{Uniqueness of the Fixed Point of TCP Models}

To prove the uniqueness of the fixed point of analytical models of TCP, we first construct a fixed point function of the TCP throughput and show that it is continuous and decreasing. We then state two theorems and prove them. We use these theorems to complete the proof of the uniqueness of the fixed point of the analytical models of TCP.

As shown in [11] and explained in [8] the throughput function given by Equation 7 can be expressed as

$$
\lambda=t\left(P_{L}, R T T\right)=\frac{1}{R T T \sqrt{\frac{2 P_{L}}{3}}+3 T_{0} \sqrt{\frac{3 P_{L}}{8}} P_{L}\left(1+32 P_{L}^{2}\right)} .
$$

This implies that for a single TCP-network sub-model pair with $N$ active TCP connections 


$$
\Lambda=N \lambda=\frac{N}{h_{2}(\Lambda) \sqrt{\frac{2 h_{1}(\Lambda)}{3}}+3 T_{0} \sqrt{\frac{3 h_{1}(\Lambda)}{8}} h_{1}(\Lambda)\left(1+32\left(h_{1}(\Lambda)\right)^{2}\right)}=F(\Lambda)
$$

where $R T T=h_{2}(\Lambda)$ and $P_{L}=h_{1}(\Lambda)$ as shown in the previous sections.

If there are $k$ TCP sub-models each of which offers $\lambda_{i}$ to the same bottleneck link, let $N_{i}$ denote the number of active TCP connections in TCP sub-model $i$. Let $D$ denote the queueing delay and $c_{i}$ refer to other components of RTT like the propagation delay which are constant for each TCP sub-model. Since $D$ is a continuous function of $E_{N}$ which in turn is a continuous function of $\Lambda, D$ is a continuous function $h_{3}(\Lambda)$. Now we have

$$
\begin{aligned}
\Lambda & =\sum_{i=1}^{k} \lambda_{i}=\sum_{i=1}^{k} N_{i} t\left(P_{L}, R T T_{i}\right)=\sum_{i=1}^{k} N_{i} t\left(P_{L}, D+c_{i}\right) \\
& =\sum_{i=1}^{k} N_{i} t\left(h_{1}(\Lambda), h_{3}(\Lambda)+c_{i}\right)=H(\Lambda) .
\end{aligned}
$$

The first derivative $F^{\prime}(\Lambda)$ is

$$
\begin{aligned}
& F^{\prime}(\Lambda)= \\
& -\left(h_{2}(\Lambda) \sqrt{\frac{2 h_{1}(\Lambda)}{3}}+3 T_{0} \sqrt{\frac{3 h_{1}(\Lambda)}{8}} h_{1}(\Lambda)\left(1+32\left(h_{1}(\Lambda)\right)^{2}\right)\right)^{-2} \times \\
& D_{\Lambda}\left(h_{2}(\Lambda) \sqrt{\frac{2 h_{1}(\Lambda)}{3}}+3 T_{0} \sqrt{\frac{3 h_{1}(\Lambda)}{8}} h_{1}(\Lambda)\left(1+32\left(h_{1}(\Lambda)\right)^{2}\right)\right) .
\end{aligned}
$$

The first derivatives of $h_{1}(\Lambda)=P_{L}$ and $h_{2}(\Lambda)=R T T=u\left(E_{N}\right)$ are positive implying that the functions $h_{1}$ and $h_{2}$ are increasing. This can be verified by the fact that when the traffic load increases the loss probability $P_{L}$ and the queuing delay $E_{N}$ both increase. Hence $F^{\prime}(\Lambda)<0$ for all possible values of $\Lambda$. This implies that the function $F(\Lambda)$ is continuous and decreasing function for $\Lambda>0\left(P_{L}>0\right)$. This can also be verified by the fact that when the loss probability and queuing delays increase the TCP throughput decreases.

Similarly it can be shown that the fixed point function $H$ used for the many TCP sub-models case is also a continuous and decreasing function of $\Lambda$.

The following statement which is based on continuous and decreasing functions may be a well known fact. However we put it as a theorem in order to easily reference it from the succeeding parts of the paper.

Theorem 3.1. A continuous decreasing function p of one variable has at most one fixed point.

Proof. The function $q(x)=x$ is an increasing function. Therefore this function and the decreasing function $p(x)$ intersect at at most one point. This in turn implies that the fixed point function $p(x)=x$ has at most one fixed point. 
Hence each of the functions $F$ and $H$ given by Equations 12 and 13 has a unique fixed point as it is also shown from the above statements that a fixed point exists (Brouwer's fixed point theorem).

Theorem 3.2. The vector valued function of two variables, $\mathbf{f}$ given by Equation 11 has a unique fixed point.

Proof. Suppose there are two fixed points $\left(P_{L_{1}}, E_{N_{1}}\right)$ and $\left(P_{L_{2}}, E_{N_{2}}\right)$. This implies that there are two fixed points $\Lambda=F(\Lambda)$ and $\Lambda^{\prime}=F\left(\Lambda^{\prime}\right)$ where $F$ is defined in Equation 12 But this is a contradiction as the function $F$ has a unique fixed point as shown above. Hence $\left(P_{L_{1}}, E_{N_{1}}\right)=\left(P_{L_{2}}, E_{N_{2}}\right)$ and the function $\mathbf{f}$ has a unique fixed point.

\section{Summary and Work in Progress}

In this paper we have shown how the FPA converges to a unique fixed point. The proof of convergence is based on a well known fixed point theorem and the uniqueness proof exploits the feedback and reliable nature of the protocol (TCP). Unlike the previous works in the literature ( [9,3]), our proof is simple and elegant and its basic principles are applicable to models of both short and long-lived TCP connections for single and multi-bottleneck links with AQM and Drop Tail routers.

We have specified (using different theorems) the conditions under which the FPA of analytical models of reliable Internet protocols like TCP and the performance of the reliable Internet protocol (TCP) itself converge to a unique fixed point.

We are extending the techniques used in this paper to prove the convergence and uniqueness of analytical models of TCP for multi-bottleneck networks with homegenous and heterogeneous (connections with different paths) TCP connections. We will also use these techniques along with some studies in the literature ( [12,13]) to further analyze the equilibrium, stability and global uniqueness issues of TCP, other reliable protocols and the Internet as a whole.

\section{Acknowledgements}

This work is supported by grant numbers 2054027 and 2677 from the South African National Research Foundation, Siemens Telecommunications and Telkom SA Limited.

\section{References}

1. F. Baccelli and D. McDonald. A square root formula for the rate of non-persistent TCP flows. In First Conference on Next Generation Internet Networks (NGI 2005), pages 171176, Rome, Italy, April 2005.

2. Kim C. Border. Fixed Point Theorems with Applications to Economics and Game Theory. Press Syndicate of the University of Cambridge, The Pitt Building, Trumpington Street, Cambridge, United Kingdom, 1985.

3. T. Bu and D. Towsley. Fixed point approximations for TCP behavior in an AQM network. In Proceedings of ACM SIGMETRICS, Cambridge, Massachusetts, USA, June 2001. 
4. M. Garetto, R. Lo Cigno, M. Meo, and M. A. Marsan. Closed queueing network models of interacting long-lived TCP flows. IEEE/ACM Transactions on Networking, 12(2):300-311, April 2004.

5. R. Gibbens, S. Sargood, C. Van Eijl, F. Kelly, H. Azmoodeh, R. Macfadyen, and N. Macfadyen. Fixed-point models for the end-to-end performance analysis of IP networks. In Proceedings of 13th ITC Special Seminar: IP Traffic Management, Modeling and Management, Monterey, California, September 2000.

6. J. J. Herings, G. van der Laan, D. Talman, and Z. Yang. A fixed point theorem for discontinuous functions. In Tinbergen Institute Discussion Papers 05-004/1, Department of Econometrics and Tinbergen Institute, Vrije Universiteit, De Boelelaan 1105, 1081 HV Amsterdam, The Netherlands, December 2004.

7. I. Kaj and J. Olsén. Stochastic equilibrium modeling of the TCP dynamics in various AQM environments. In International Symposium on Performance Evaluation of Computer and Telecommunication Systems (SPECTS'02), San Diego, USA, July 2002.

8. C. T. Kelly. Engineering flow controls for the Internet, A dissertation submitted to the Cambridge University for the degree of Doctor of Philosophy. PhD thesis, LABORATORY FOR COMMUNICATION ENGINEERING, Department of Engineering, Cambridge University, 2004.

9. M. Meo, M.Garetto, M. A. Marsan, and R. Lo Cigno. On the use of fixed point approximations to study reliable protocols over congested links. In Globecom, San Francisco, USA, December 2003.

10. Jorgen Olsén. Stochastic Modeling and Simulation of the TCP Protocol. PhD thesis, Department of Mathematics, Uppsala University, Box 480, SE-751 06 Uppsala, Sweden, 2003.

11. J. Padhye, V. Firoiu, D. Towsley, and J. Kurose. Modeling TCP Reno performance: a simple model and its empirical validation. IEEE/ACM Transactions on Networking, 8(2):133-145, April 2000.

12. A. Tang, J. Wang, S. H. Low, and M. Chiang. Network equilibrium of heterogeneous congestion control protocols. In IEEE INFOCOM, Miami, FL USA, March 2005.

13. J. Wang, L. Li, S. H. Low, and J. C. Doyle. Cross-layer optimization in TCP/IP networks. IEEE/ACM Transactions on Networking, 13(3):568-582, June 2005. 